\title{
Galactic Cosmic Rays: The first detection of TeV gamma-rays from Red Dwarfs
}

\author{
V.G. Sinitsyna ${ }^{1, *}$, V.Y. Sinitsyna ${ }^{1}$, Yu.I. Stozhkov ${ }^{1}$ \\ ${ }^{1}$ P. N. Lebedev Physical Institute, Russian Academy of Sciences
}

\begin{abstract}
The present point of view on the sources of cosmic rays in Galaxy considers explosions of supernovae as sources of these particles up to energies of $10^{17} \mathrm{eV}$. However, the experimental data obtained with Pamela, Fermi, AMS-02 spectrometers requires the existence of nearby sources of cosmic rays at distances less then 1 kpc from the solar system. These sources could explain such experimental data as the growth of the ratio of galactic positrons to electrons with increasing energy, the complex dependence of the exponent of the proton and alpha spectra from the energy of these particles, the appearance of an anomaly component in cosmic rays. We consider active dwarf stars as possible sources of galactic cosmic rays in the energy range up to $10^{14} \mathrm{eV}$. These stars produce powerful stellar flares. The generation of high-energy cosmic rays has to be accompanied by high-energy gamma-ray emission. Here we present the SHALON long-term observation data aimed at searching for gamma-ray emission above $800 \mathrm{GeV}$ from active red dwarf stars. The data obtained during more than 10 years observations of the dwarf stars V962 Tau, V780 Tau, V388 Cas and V1589 Cyg were analyzed. The high-energy gamma-ray emission in the TeV energy range, mostly of the flaring type from the sources mentioned above, was detected. This result confirms that active dwarf stars are also the sources of high-energy galactic cosmic rays.
\end{abstract}

\section{Introduction}

Supernova Remnants (SNRs) have long been considered as unique candidates for cosmic-ray sources $[1,2]$. Recent observations of SNRs in $\mathrm{TeV} \gamma$-rays are making clear the origin of cosmic rays in these objects. However, the experimental data obtained with the Pamela, Fermi, AMS02 spectrometers requires the existence of nearby sources of cosmic rays at distances $<1 \mathrm{kpc}$ from the solar system. These sources could explain such experimental data as the growth of the ratio of galactic positrons to electrons with increasing energy (Fig. 1), the complex dependence of the exponent of the proton and alpha spectra from the energy of these particles, the appearance of an anomaly component in cosmic rays. We consider active dwarf stars as possible sources of galactic cosmic rays in the energy range up to $10^{14} \mathrm{eV}$. These stars produce powerful stellar flares. The generation of high-energy cosmic rays has to be accompanied by high-energy $\gamma$-ray emission. Here we present the SHALON long-term observation data aimed to search for $\gamma$-ray emission above $800 \mathrm{GeV}$ from active red dwarf stars. The data obtained during more than 10 years observations of the dwarf stars V962 Tau, V780 Tau, V388 Cas and V1589 Cyg were analyzed.

\section{Observations of Red dwarfs}

In the studies of flaring stars it was shown, that the frequency of flaring is uncertain [3, 4], so to search for TeV $\gamma$-ray emission from these objects we chose objects that we

\footnotetext{
*e-mail: sinits@sci.lebedev.ru
}

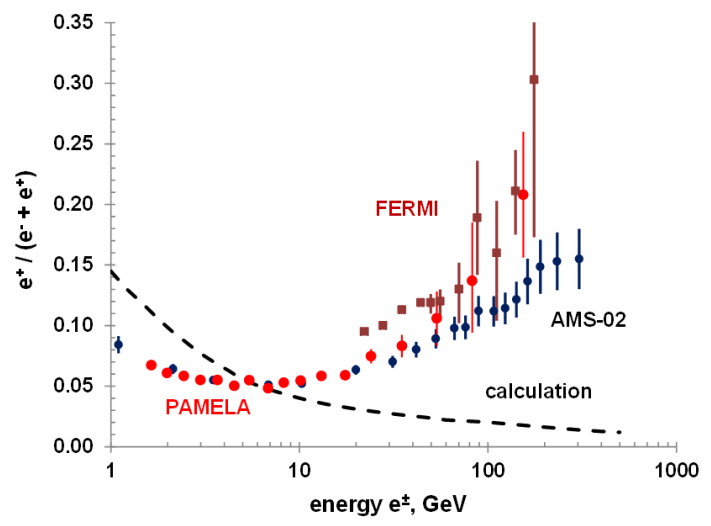

Figure 1. The positron fraction measured by PAMELA, AMS, Fermi LAT [5-7].

have collected data from during different long-term observations. In accordance with the long-term program studying galactic $\gamma$-ray sources, more than ten-year-long observations of Tycho's SNR, Crab Nebula and Cyg X-3, are being carried out in the SHALON experiment $[8,9]$. During these observations the SHALON field of view contains the red dwarfs listed above as it is located at angular distances less than $\sim 5^{\circ}$ from observed TeV $\gamma$-ray sources. So due to the large telescopic field of view $>8^{\circ}$ the observations of investigated objects are naturally followed by the observations of these flaring stars. The observations and data processing were performed using the standard SHALON 

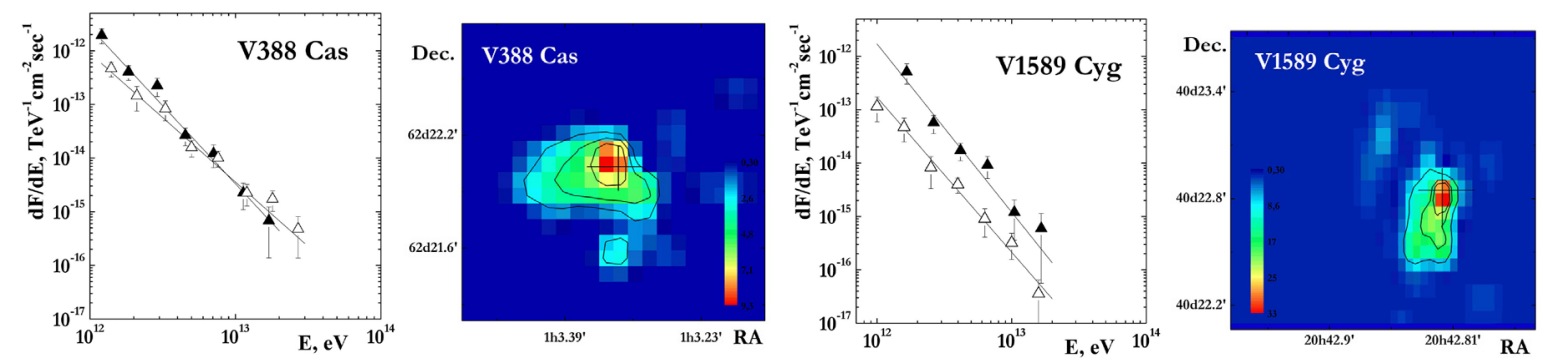

Figure 2. V388 Cas and V1589 Cyg differential spectra and images by SHALON (see text).

technique $[10,11]$. Results on each of the red dwarfs are presented below.

\subsection{V388 Cas}

Tycho's SNR was observed with SHALON during 123 hours in the period from 1996 to 2010 [12, 13]. All observations were made during moonless nights with zenith angles from $16^{\circ}$ to $35^{\circ}$. During the observations of Tycho's SNR the SHALON field of view contains V388 Cas as it is located at $\sim 4.5^{\circ}$ South from Tycho's SNR. So, due to the large telescopic field of view $\left(>8^{\circ}\right)$, observations of Tycho's SNR are naturally followed by observations of V388 Cas flaring star. V388 Cas is a UV Cet type star [14] at distance $\sim 10$ pc. It is also in the ROSAT catalogue with flux $\sim 1.7 \times 10^{-12} \mathrm{erg} \mathrm{cm}^{-2} \mathrm{~s}^{-1}$ and luminosity of $10^{28.3} \mathrm{erg} / \mathrm{s}$ [14]. V388 Cas as a source accompanying Tycho's SNR was observed with the SHALON telescope in the period 1996 to 2010 for a total of 93 hours. The $\gamma$-ray source associated with V388 Cas was detected above $1 \mathrm{TeV}$ with a statistical significance $6.8 \sigma$ determined by Li \& Ma [15] and with average $\gamma$-ray flux: $I_{V 388 \text { Cas }}(>1 \mathrm{TeV})=(0.84 \pm 0.21) \times 10^{-12} \mathrm{~cm}^{-2} \mathrm{~s}^{-1}$.

The signal significance for this source is much less than that for the source with similar flux and spectrum index obtained in the same observation hours because of a smaller collection field of view relative to the standard procedure of the SHALON experiment. After processing Tycho's SNR observation data first by selection criteria associated with Tycho's SNR and then with V388 Cas we found that less than $1 \%$ of showers are common for both sources. Recognition of the source for each of the common showers is performed by analysing the angular distance of arrival directions of these showers and the source coordinates. This didn't change the average flux of Tycho's SNR. The shape of SHALON's average differential spectrum of $\gamma$-rays from V388 Cas (Fig. 2, $\Delta$ ) in the energy range from 0.8 to $25 \mathrm{TeV}$ fits well to a soft power law: $d N / d E=(0.91 \pm 0.25) \times 10^{-12} \times\left(E_{\gamma} / 1 \mathrm{TeV}\right)^{-2.52 \pm 0.15}$ with the $\chi^{2} / D o f=1.23$ where the degree of freedom $D o f=6$. The emission map for V388 Cas is shown in Fig. 2. During long-term observations V388 Cas appeared as a source with flux variations and seemed to be detected during the flares. A flaring spectrum: $d N / d E_{\text {Flare }}=$ $(2.7 \pm 0.15) \times 10^{-12} \times\left(E_{\gamma} / 1 \mathrm{TeV}\right)^{-2.91 \pm 0.18}($ Fig. $2, \mathbf{\Delta})$. The integral fluxes of V388 Cas averaged over each year observation period are shown in Fig. 4.

\subsection{V1589 Cyg}

In accordance with the program on long-term studies of the microquasar Cygnus X-3 at very high energies, obser- vations of the Cygnus Region and its objects, including V1589 Cyg, as well as TeV J2032+4130 and $\gamma$ Cygni SNR are being carried out with SHALON [9, 11, 18]. V1589 Cyg is a flare star of the M class. Its luminosity was detected to vary from 13 to 15 [3] and the distance was estimated to be 23 - 32 pc. V1589 Cyg demonstrates flares with durations from minutes to hours [3].

V1589 Cyg is located at a distance of $\sim 2^{\circ}$ west from Cyg X-3. So due to the large telescopic field of view $>8^{\circ}$ the observations of Cygnus- $X$ are naturally followed by tracking V1589 Cyg. As a result, V1589 Cyg as a source accompanying Cyg X-3 have been systematically observed with the SHALON telescope (since 1995 up to 2016) during clear moonless nights at zenith angles from $5^{\circ}$ to $34^{\circ}$ for a total of 303.5 hours. With the data processing, V1589 Cyg was detected above $0.8 \mathrm{TeV}$ by SHALON with an average integral flux: $I_{V 1589 C_{y g}}(>$ $0.8 \mathrm{TeV})=(0.13 \pm 0.019) \times 10^{-12} \mathrm{~cm}^{-2} \mathrm{~s}^{-1}$. V1589 Cyg was detected above $0.8 \mathrm{TeV}$ by SHALON with a statistical significance $6.5 \sigma$ determined by the Li \& Ma [15] method. The signal significance for this source is less than one for the source with similar flux and spectrum index obtained in the same observation hours because of a smaller collection field of view relative to the standard procedure of the SHALON experiment. Corrections for the effective field of view were made. After processing the Cyg X-3 observation data first by selection criteria associated with Cyg X-3 and then with V1589 Cyg we found that less than $2 \%$ of showers are common for both sources. Recognition of the source for each of the common showers is performed by analysing the angular distance of arrival directions of these showers and the source coordinates. As a result, less than $1 \%$ of Cyg X-3 showers were recognized to be V1589 Cyg showers. This didn't change the average flux of Cyg X-3. During long-term observations V1589 Cyg appeared as a source with variable flux. The flaring spectrum was also extracted and is shown in Fig. $2 \boldsymbol{\Delta}$. The shape of the SHALON differential spectrum of $\gamma$-rays from V1589 Cyg in the energy range from 0.8 to $35 \mathrm{TeV}$ fits well to a soft power law (see Fig. $2 \triangle) . d N / d E=(0.15 \pm 0.05) \times 10^{-12} \times\left(E_{\gamma} / 1\right.$ $\mathrm{TeV})^{-2.91 \pm 0.18}$ with a $\chi^{2} /$ Dof $=0.89$ where degree of freedom $D o f=5$. An emission map for V1589 Cyg is shown in Fig. 2. A flaring spectrum: $d N / d E_{\text {Flare }}=$ $(1.7 \pm 0.45) \times 10^{-12} \times\left(E_{\gamma} / 1 \mathrm{TeV}\right)^{-3.15 \pm 0.29}$. The light curve of V1589 Cyg during the observations from 1995 to 2016 is shown in Fig. 4. The integral fluxes averaged over each year's observation period are shown. 

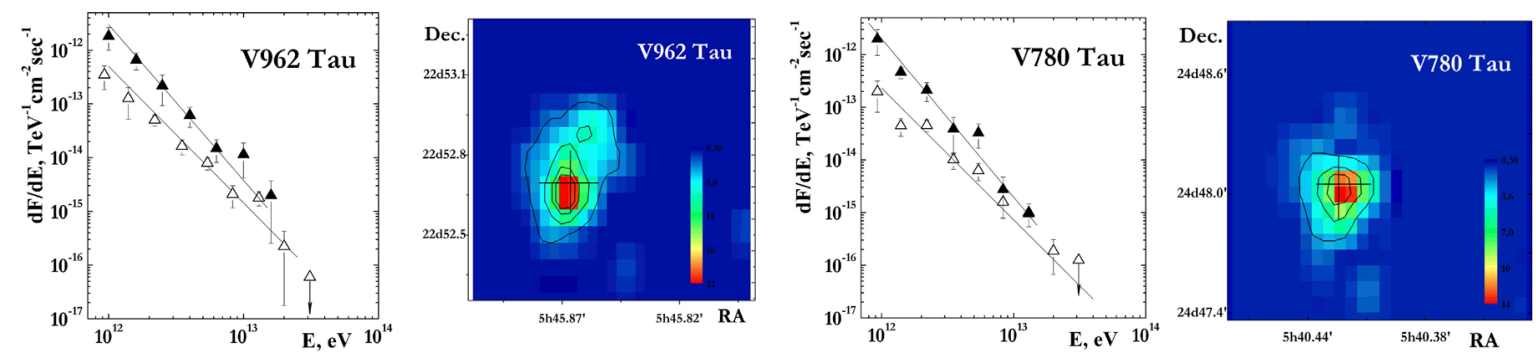

Figure 3. V962 Tau and V780 Tau differential spectra and images by SHALON (see text).

\subsection{V962 Tau and V780 Tau}

Observations of the Crab Nebula were accompanied with tracking through the telescopic field of view of two flaring stars: V962 Tau and V780 Tau. V962 Tau and V780 Tau are the flaring type of UV Cet type with spectrum red star class $[14,16]$ at distances about $10 \mathrm{pc}$. Luminosities, $M_{V}$, detected varied from 13 to 15 . V780 Tau is also visible by ROSAT with a flux $\sim 3.6 \times 10^{-13} \mathrm{erg} \mathrm{cm}^{-2} \mathrm{~s}^{-1}$ and luminosity $10^{27.6} \mathrm{erg} / \mathrm{s}$ [14].

V780 Tau is located at a distance of $\sim 3^{\circ}$ north from the Crab and V962 Tau is $2.5^{\circ}$ East from the Crab. These stars are $\sim 4^{\circ}$ separated from each other. As a result, V780 Tau and V962 Tau as sources accompanying the Crab have been systematically observed with SHALON since 1994 $[8,17]$ during clear moonless nights. These flaring stars as sources accompanying the Crab were observed with SHALON in the period 1994 to 2014 for a total of 125.2 hours during clear moonless nights at zenith angles from $15^{\circ}$ to $35^{\circ}$. With the data processing, SHALON detected V780 Tau and V962 Tau above $0.8 \mathrm{TeV}$ with an average integral flux: $I_{V 780 \mathrm{Tau}}(>1 \mathrm{TeV})=(0.23 \pm 0.03) \times 10^{-12}$ $\mathrm{cm}^{-2} \mathrm{~s}^{-1}$ and $I_{V 962 \mathrm{Tau}}(>1 \mathrm{TeV})=(0.39 \pm 0.04) \times 10^{-12}$ $\mathrm{cm}^{-2} \mathrm{~s}^{-1}$. Emission maps for V962 Tau and V780 Tau are shown in Fig. 3.

V780 Tau was detected by SHALON above $0.8 \mathrm{TeV}$ with a statistical significance $6.1 \sigma$ determined by the $\mathrm{Li} \&$ Ma method [15]. The signal significance for this source is also less than one for the source with a similar flux obtained in the same observation hours because of a smaller collection field of view relative to the standard experimental procedure. After processing the Crab observation data first by selection criteria associated with the Crab and then with V780 Tau we found that less than $1 \%$ of showers are common to both sources. Recognition of sources for each of the common showers is performed by analysing the angular distance of arrival direction of these showers and source coordinates. As a result, less than $\sim 0.4 \%$ of Crab showers were recognized to be V780 Tau showers. This didn't change the average flux of the Crab. In observations since 1994, V780 Tau was found to be variable. The flaring spectrum was also extracted (see Fig. $3 \mathbf{\Delta}$ ). The shape of the SHALON differential spectrum of $\gamma$-rays from V780 Tau in the energy range from 0.8 to $20 \mathrm{TeV}$ fits well to a power law: $d N / d E=(0.21 \pm 0.08) \times 10^{-12} \times\left(E_{\gamma} / 1 \mathrm{TeV}\right)^{-2.51 \pm 0.15}$ with the $\chi^{2} / D o f=1.31$ where the degree of freedom Dof $=5$ (see Fig. $3 \triangle$ ). A flaring spectrum: $d N / d E_{\text {Flare }}=$ $(2.0 \pm 0.15) \times 10^{-12} \times\left(E_{\gamma} / 1 \mathrm{TeV}\right)^{-3.01 \pm 0.21}$ (see Fig. $\left.3 \mathbf{\Delta}\right)$.

V962 Tau was detected above $0.8 \mathrm{TeV}$ by SHALON with a statistical significance $7.7 \sigma$ determined by the Li \&
Ma method [15]. The signal significance for this source is also less than one for a source with similar flux obtained in the same observation hours because of a smaller collection field of view relative to the standard experiment procedure. After processing the Crab observation data first by selection criteria associated with the Crab and then with V780 Tau we found that there are no showers common for both sources. V962 Tau was also found to be variable. The differential spectrum of $\gamma$-rays from V962 Tau in the energy range from 0.8 to $20 \mathrm{TeV}$ fits well to a power law: $d N / d E=(0.40 \pm 0.17) \times 10^{-12} \times\left(E_{\gamma} / 1 \mathrm{TeV}\right)^{-2.54 \pm 0.15}$ with the $\chi^{2} /$ Dof $=0.87$ where Dof $=6$ (see Fig. $2 \Delta$ ). The flaring spectrum: $d N / d E_{\text {Flare }}=(2.8 \pm 0.45) \times 10^{-12} \times\left(E_{\gamma} / 1\right.$ $\mathrm{TeV})^{-2.95 \pm 0.22}$ is shown by $\boldsymbol{\Delta}$ in Fig. 3 . Both these active red dwarfs display a behavior with variable fluxes at $\mathrm{TeV}$ energies. Light curves of V780 Tau, V962 Tau obtained during the long-term observations are presented in Fig. 4 .

\section{Discussion}

During long-term observations, red dwarfs appeared as sources with variable $\mathrm{TeV} \gamma$-ray fluxes up to energy $\sim 10$ $\mathrm{TeV}$ and seemed to be detected mostly during flares. The light curves of V388 Cas, V780 Tau, V962 Tau and V1589 Cyg at TeV energies obtained in the long-term SHALON observations are shown in Fig. 4. The integral fluxes averaged over each year's observation period are shown. M-Dwarf stars are characterized by flaring events which occur frequently with flare duration varying in time but for a total duration of $\sim 10^{4} \mathrm{sec}[3,4]$. Very high energy $\gamma$-ray fluxes presented here demonstrate that these objects produce the flares of $\sim 10^{33}-10^{35}$ ergs.

An anomalous effect of the dependence on the energy of the ratio, $r$, of the flux of positrons to the total flux of Galactic positrons and electrons was discovered in the PAMELA experiment and then confirmed in AMS and Fermi LAT. In theory, this ratio should decrease with increasing particle energy. However, the experiment showed that in the energy range of $0.1 \mathrm{GeV}<E<5 \mathrm{GeV}$, the value of $\mathrm{r}$ decreases and its growth is then observed beginning from $E>5 \mathrm{GeV}$ up to $\sim 150-300 \mathrm{GeV}$. The explanation for this effect is based on the thesis that the sources of GCRs in the energy range $E<10^{14} \mathrm{eV}$ are not only supernovae but also active dwarf stars that make up more than $95 \%$ of all the stars in our Galaxy.

Indeed, active dwarf stars can be considered as sources of Galactic Cosmic Rays as stellar flares of Dwarf Stars provide the observed energy of GCR which is about $W_{G C R} \sim 2 \times 10^{54} \mathrm{erg}$ up to energies of $10^{13}-10^{14} \mathrm{eV}$ [19]. Among the huge variety of stars in our Galaxy in which there are $\sim 2 \times 10^{11}$ stars of mass equal to Sun, more than 


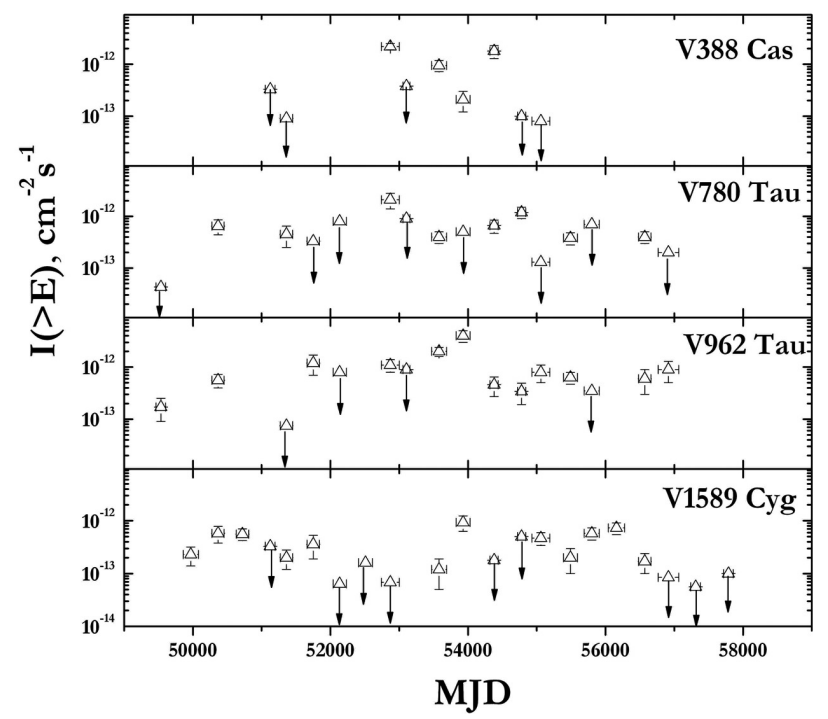

Figure 4. Light curves of V388 Cas, V780 Tau, V962 Tau, V1589 Cyg at TeV energies from long-term SHALON observations.

95\% are dwarf stars of G-M spectral classes [14]. Suppose that $10 \%$ of the dwarf stars in our Galaxy produce stellar flares with frequency of flares of about 36 year $^{-1}$ and the average energy released in stellar flares about $10^{35}$ ergs one finds that $W \sim 1.4 \times 10^{54}$ erg which is comparable to the accepted estimations of GCR energy, $W_{G C R}$, in the Galactic disk.

During powerful flares from active dwarf stars, highenergy protons are generated with a maximum energy of $E<10^{14} \mathrm{eV}$. Some of these accelerated protons escape into the interstellar medium. Others enter the photosphere of the star and undergo nuclear interactions with its matter. In the course of these interactions, $\pi^{\circ}$ and $\pi^{ \pm}$are formed, while positrons and electrons are produced during $\pi^{ \pm}$to $\mu^{ \pm} e^{ \pm}$decay. An additional source of positrons is the decay of neutral pions into two $\gamma$-rays and the subsequent formation of $\left(e^{+} e^{-}\right)$pairs by these $\gamma$-rays. Such a process is observed on the Sun during large solar flares (the Sun is a yellow dwarf). Positrons and electrons from stellar flares with $E>5 \mathrm{GeV}$ escape into the interstellar medium from a flare region with strong magnetic fields. Thus, there is an additional source of $e^{+}$that explains the anomalous PAMELA effect. It should be noted that most of the electrons (and protons) are accelerated during the stellar flare and the addition of another $e^{-}$flux via the mechanisms of $\pi^{\circ}$ and $\pi^{-}$decays constitutes only a small part of the main flux. The additional source of $e^{+}$, however, provides the main flux of these particles in the Galaxy up to energies of $E \sim(300-400) \mathrm{GeV}$. Calculations show that this mechanism explains the increase in the ratio of positrons to the total flux of Galactic positrons and electrons in the energy range of $E>5 \mathrm{GeV}$.

\section{Conclusion}

The results of long-term observations by the SHALON mirror Cherenkov telescope of M-Dwarf stars V388 Cas, V780 Tau, V962 Tau and V1589 Cyg are presented. Very high energy gamma-ray emission in the range of $1-10$ $\mathrm{TeV}$, mostly of the flaring type from these red dwarfs, are detected. The emission is characterized with a soft powerlow spectrum with $\sim 2.6 \div 3.2$. This result confirms that active dwarf stars are also the sources of high energy cosmic rays. These sources could explain such experimental data as the growth of the ratio of galactic positrons to electrons with increasing energy observed by the PAMELA and AMS spectrometers.

\section{References}

[1] E.G. Berezhko, G.F. Krymskii Usp. Fiz. Nauk 154(1), 49 (1988)

[2] S.P. Reynolds Ann. Rev. Astron. Astrophys. 46, 89 (2008)

[3] B.R. Pettersen et al., Astrophysics 29(1), 67 (1988)

[4] S. Ohm, C. Hoischen MNRAS 474, 1335 (2018)

[5] O. Adriani et al. Nature, 458(7238), 607 (2009)

[6] M. Ackermann et al. Phys. Rev. Lett. 108, 011103 (2012)

[7] M. Aguilar et al. PRL 110, 141102 (2013)

[8] V.G. Sinitsyna Nuovo Cim. 19C, 965 (1996)

[9] V.G. Sinitsyna, in Proc. of the 16th ECRS, Ed. by J. Medina (Univ. de Alcala, Spain, 1998), p. 383.

[10] V.G. Sinitsyna et al.,ASR 62(10), 2845 (2018)

[11] V.G. Sinitsyna, V.Yu. Sinitsyna, Astron. Lett. 44(3), 162 (2018)

[12] V.G. Sinitsyna, Towards a Major Atmospheric Cherenkov Detector-V, (Kruger Park, 1997) ed O.C. deJager (Potchefstroom: Westprint) p 136

[13] V.G. Sinitsyna, V.Yu. Sinitsyna, Astron. Lett. 37(9), 621 (2011)

[14] R.E. Gershberg et al., Astron. Astrophys. Suppl. Ser. 139, 555 (1999)

[15] T.-P. Li, Y.-Q. Ma, Astrophys. J. 272, 317 (1983)

[16] I.V. Hatsunzev, Variable Stars 22(3), 431 (1986)

[17] V.G. Sinitsyna et al., J. Phys.: Conf. Ser. 718, 052045 (2016)

[18] V.G. Sinitsyna, V.Yu. Sinitsyna, Bull. of the Lebedev Phys. Inst. 40(5), 113 (2013)

[19] Yu.I. Stozhkov, Bull. of RAS, ser. Physics 75(3) 323 (2011) 